\section{Reliable method for generating double- stranded DNA vectors containing site-specific base modifications}

\author{
Damien Brégeon and Paul W. Doetsch \\ Emory University School of Medicine, Atlanta, GA, USA
}

BioTechniques 37:760-766 (November 2004)

Cells of all living organisms are continuously exposed to physical and chemical agents that damage DNA and alter the integrity of their genomes. Despite the relatively high efficiency of the different repair pathways, some lesions remain in DNA when it is replicated or transcribed. Lesion bypass by DNA and RNA polymerases has been the subject of numerous investigations. However, knowledge of the in vivo mechanism of transcription lesion bypass is very limited because no robust methodology is available. Here we describe a protocol based on the synthesis of a complementary strand of a circular, single-stranded DNA molecule, which allows for the production of large amounts of double-stranded DNA containing a lesion at a specific position in a transcribed sequence. Such constructs can subsequently be used for lesion bypass studies in vivo by RNA polymerase and to ascertain how these events can be affected by the genetic background of the cells.

\section{INTRODUCTION}

The genomes of all organisms are constantly exposed to chemical and physical agents that damage the DNA molecule. Non-bulky DNA lesions do not generally block the transcription complex but have often altered coding properties, which results in the insertion of a noncomplementary nucleotide opposite the lesion, leading to transcriptional mutagenesis (TM; References $1-3)$. The generation of mutated transcripts through TM could also have many biological outcomes $(1,4)$, but this has not been extensively analyzed in vivo because of the lack of robust techniques allowing for its study. Consequently, the need for reliable methodologies to study lesion bypass by RNA polymerase within cells is an important issue. Such methodologies would rely on the construction of double-stranded closed circular DNA (ccDNA), containing a DNA lesion at a specific position in a transcribed sequence, which would be transformed or transfected into cells.

One of the major issues is that the synthetic product has to be produced in large quantities because it cannot be propagated in cells to avoid any replication-related events. A technique that might be used is PCR amplification of a whole plasmid with a primer containing a damaged base (5), but the high-fidelity archaebacterial DNA polymerases used in this procedure are inhibited by uracil and potentially other DNA lesions (6). Another extensively utilized procedure is the replacement of a small restriction fragment of the plasmid with a synthetic duplex DNA segment containing a DNA lesion at a specific site. The efficiency of this method is limited, and only a small percentage corresponds to ccDNA (7). A more efficient derivative of this technique, the so-called gapped-duplex method (8), is only suitable for transcription lesion bypass analysis in bacteria because the DNA adduct is placed in a transcribed sequence of the M13mp7L2 bacteriophage genome. The last technique that can be used for site-directed DNA damage placement involves enzymatic extension by a DNA polymerase of a $5^{\prime}$ phosphorylated oligonucleotide primer hybridized to a circular single-stranded DNA (ssDNA). CcDNA molecules are formed by ligation of the newly synthesized strand with a DNA ligase
(9). The yield of synthetic ccDNA produced with this procedure is only a few percent (10). In this study, we report that several modifications made to the previously described protocols greatly enhanced yields of DNA damage-containing products.

\section{MATERIALS AND METHODS}

Unless otherwise specified, all the products used for the procedures described below were obtained from Sigma (St. Louis, MO, USA). Two procedures for the production of ssDNA were compared and, for both of them, phage suspensions were prepared as follows. A single colony of the bacterial strain DH12S carrying the pBESTlucf1-Stop phagemid (11) was resuspended in $5 \mathrm{~mL}$ of Luria Bertani (LB) broth containing ampicillin $(100 \mu \mathrm{g} / \mathrm{mL})$ and grown overnight. This overnight culture was diluted 2000 -fold in $200 \mathrm{~mL}$ of LB containing ampicillin and incubated at $37^{\circ} \mathrm{C}$ for $3 \mathrm{~h}$ with shaking $(250$ rpm). M13KO7 helper phage $\left[2 \times 10^{10}\right.$ phage-forming units (pfus)] was then added to the media, and incubation was continued for $2 \mathrm{~h}$ prior to the addition of kanamycin $(50 \mu \mathrm{g} / \mathrm{mL})$. The infected cells were incubated at $37^{\circ} \mathrm{C}$ for an additional 18 to $24 \mathrm{~h}$ with shaking. The culture was centrifuged at $16,000 \times g$ for $15 \mathrm{~min}$ at $4^{\circ} \mathrm{C}$, and the supernatant was filtered through a $0.2-\mu \mathrm{m}$ filter. From this step, ssDNA was prepared either as previously described (10) or as follows. Phage particles were precipitated at $4^{\circ} \mathrm{C}$ by the addition of $6 \mathrm{~g} \mathrm{NaCl}$ and $10 \mathrm{~g}$ polyethylene glycol (PEG)-8000. The addition of PEG- 8000 to the media was made $1 \mathrm{~g}$ at a time at $4^{\circ} \mathrm{C}$ with slow stirring and allowing for the complete dissolution of PEG between each addition. Phage precipitate was centrifuged at $16,000 \times g$ for $20 \mathrm{~min}$ at $4^{\circ} \mathrm{C}$, and the pellet was resuspended into $5 \mathrm{~mL}$ of TE (Tris-EDTA) and $50 \mu \mathrm{L}$ $10 \%$ sodium dodecyl sulfate (SDS). DNA was separated from proteins by 4 phenol:chloroform:isoamyl alcohol (PCIA) $(25: 24: 1)$ extractions and precipitated with ethanol. The DNA pellet was then solubilized into a $550 \mathrm{mM}$ $\mathrm{NaCl} / 50 \mathrm{mM}$ MOPS (pH 7.0) solution. To purify the ssDNA, the DNA solution was passed through a QIAGEN-tip 
500 (Qiagen, Valencia, CA, USA) following the manufacturer's instructions, except that the final ssDNA elution was made with $15 \mathrm{~mL}$ QM $(1 \mathrm{M} \mathrm{NaCl}, 50$ mM MOPS, pH 7.0, 4 M urea, 30\% $\mathrm{EtOH} 30)$. The ssDNA was then precipitated and resupended in $500 \mu \mathrm{L}$ TE. DNA concentration was quantified by spectrophotometry $\left(\mathrm{A}_{260}\right)$.

For the second-strand synthesis, $40 \mu \mathrm{g}$ ssDNA were mixed in $1 \times$ standard saline citrate (SSC; $150 \mathrm{mM} \mathrm{NaCl}$, $15 \mathrm{mM}$ sodium citrate, $\mathrm{pH} 7.0$ ) with a 12 -fold molar excess ( $320 \mathrm{pmol})$ of the following primer $\left(5^{\prime}\right.$-phos-CGATTCCAATTAAGCGGGGGCCACCTGATATCCTTXGTATTTAAT-3'; IDT, Coralville, IA, USA) where $\mathrm{X}$ is A for the stop construct and 8-oxoguanine (8OG) for the $80 G$ construct. The tube containing this mixture was placed in water at $95^{\circ} \mathrm{C}$ and allowed to cool to room temperature. When specified, this mixture was filtered through Microcon Montage ${ }^{\circledR}$-PCR filter units (Millipore, Billerica, MA, USA). In order to avoid membrane saturation, it was necessary to divide the annealing reaction into four samples of equivalent volume. The DNA polymerization reaction was carried out in a $300-\mu \mathrm{L}$ reaction mixture containing the ssDNA/oligonucleotide hybrid, $10 \mathrm{mM}$ Tris- $\mathrm{HCl}, \mathrm{pH} 7.9,50$ $\mathrm{mM} \mathrm{NaCl}, 10 \mathrm{mM} \mathrm{MgCl} 2,1 \mathrm{mM}$ dithiothreitol (DTT), $50 \mu \mathrm{g} / \mathrm{mL}$ bovine serum albumin (BSA), $600 \mu \mathrm{M}$ each of dATP, dCTP, dTTP, and dGTP, $1 \mathrm{mM}$ ATP, 30 U T4 DNA polymerase, and 60 $\mathrm{U}$ of T4 DNA ligase. When specified, PEG-8000 was added to the reaction at a final concentration of 7.5\%. To stabilize the initial duplex between the template DNA and the primer, the reaction was first incubated for $5 \mathrm{~min}$ on ice and then for $5 \mathrm{~min}$ at room temperature. Polymerization and ligation reactions were then completed at $37^{\circ} \mathrm{C}$ for $2 \mathrm{~h}$ or overnight.

Closed circular molecules were purified from a $0.6 \%$ agarose gel containing $0.3 \mu \mathrm{g} / \mathrm{mL}$ ethidium bromide. Under these conditions, ccDNA migrates as supercoiled DNA and can be resolved from single-stranded closed circular and nicked double-stranded plasmids. Several methods were compared for the extraction of DNA from the agarose gel. For regular agarose gels, these methods included the use of the QIAquick ${ }^{\circledR}$ Gel Extraction Kit (Qiagen) with or without the optional addition of an isopropanol step and the Ultrafree ${ }^{\circledR}$-DA DNA Extraction From Agarose Gels Kit (Millipore). The two other procedures used were based on digestion of low melting point agarose with $\beta$-agarase (New England Biolabs, Beverly, MA, USA), followed either by DNA precipitation or by purification with Microcon YM100 Columns (Millipore).

The presence of a single DNA lesion in the construct was assessed by treating the $80 \mathrm{OG}$ construct with formamidopyrimidine (Fapy)-glycosylase (FPG; New England Biolabs). As a control, the stop construct was treated in parallel with the same enzyme.

\section{RESULTS AND DISCUSSION}

The method of construction of a closed circular double-stranded DNA plasmid containing a DNA lesion at a defined site is based on a site-directed mutagenesis procedure using ssDNA as a template (9). In order to achieve good yields of these types of constructs, we first identified a procedure allowing the production of ssDNA in large quantities.

Two procedures were compared for the production of ssDNA from DH12S cells carrying the pBESTluc-f1-Stop phagemid. The best procedure for the preparation of ssDNA is based on the slow precipitation of phage particles followed by PCIA extraction. Contaminating double-stranded DNA, polysaccharides, and protein traces were eliminated by passing the solution through an anion-exchange resin with elution of the ssDNA with a specific buffer. With this procedure, the amount of ssDNA obtained from a $200 \mathrm{~mL}$ phage preparation was $186 \mu \mathrm{g} \pm 14$ ( \pm SEM) of ssDNA instead of $40 \mu \mathrm{g} \pm 2$ with a previously described method (10). It appeared that production of pure ssDNA in large quantities is more efficiently achieved with this procedure.

For the synthesis of the damagecontaining strand, a phosphorylated oligonucleotide, complementary to the (+) strand of the phagemid containing a DNA lesion at a specific residue, is annealed to the circular ssDNA. This oli- 
Table 1. Comparison of Different Gel Extraction Methods

\begin{tabular}{|lccc|}
\hline Method & $\begin{array}{c}\text { DNA Amount Recovered } \\
(\mathbf{n g})(\overline{\times} \pm \text { SEM) }\end{array}$ & $\begin{array}{c}\text { Purity }\left(\mathbf{A}_{\mathbf{2 6 0}} / \mathbf{A}_{\mathbf{2 8 0}}\right)^{\mathbf{a}} \\
(\overline{\times} \pm \text { SEM) }\end{array}$ & $\begin{array}{c}\text { Relative Yield } \\
(\%)\end{array}$ \\
\hline QIAquick + isopropanol & $140 \pm 20.2$ & $2.2 \pm 0.40$ & 30.3 \\
QIAquick - isopropanol & $128 \pm 11.7$ & $1.8 \pm 0.17$ & 27.8 \\
UltraFree DA & $241 \pm 13.4$ & $1.4 \pm 0.12$ & 52.1 \\
$\beta$-agarase + precipitation & $462 \pm 32.1$ & $1.9 \pm 0.02$ & 100.0 \\
$\beta$-agarase + YM100 & $224 \pm 8.1$ & $1.7 \pm 0.03$ & 48.5 \\
Samples (10 $\mu$ L aliquots of a polymerization/ligation reaction) were run on 0.6\% agarose gels (column-based \\
$\begin{array}{l}\text { kits) or 0.6\% low melting point gels ( } \beta \text {-agarase digestion methods). +, with; --, without. } \\
\text { aQuantified by ultraviolet (UV) spectrophotometry (absorbance at 260 nm). }\end{array}$ \\
bNormalized to the $\beta$-agarase + precipitation method. \\
\hline
\end{tabular}

gonucleotide is subsequently extended with T4 DNA polymerase for the synthesis of the second strand. The ccDNA molecules are then formed by ligation of the newly synthesized strand with T4 DNA ligase. The amount of ccDNA produced with this method in previously described conditions (12) is very low (Figure 1, lane 1).

It seemed likely that one of the limiting steps was the reaction time used. After $2 \mathrm{~h}$ of incubation with DNA polymerase and DNA ligase, the yield of double-stranded circular molecule is very low, and there is no detectable product corresponding to the closed circular form (Figure 1, lane 1). By extending the incubation to $16-18 \mathrm{~h}$, larger amounts of circular DNA (closed or relaxed) are detected on an agarose gel (Figure 1, lanes 1, 3, 5, and 7 versus lanes 2, 4, 6, and 8). prove was the ligation efficiency of the reaction. It was possible to generate a significant amount of circular relaxed double-stranded DNA with 16-18 h of polymerization/ligation reaction
One of the steps we wanted to im-

(Figure 1, lanes 2, 4, 6, and 8). It is known that moderate concentrations of monovalent cations can inhibit the activity of the T4 DNA ligase (13) and may also decrease the efficiency of the T4 DNA polymerase (14). The annealing of the oligonucleotide containing a DNA lesion with ssDNA was carried out in the presence of $1 \times \mathrm{SSC}$ so that the addition of the annealing solution to the polymerization reaction significantly increases the $\mathrm{Na}^{+}$concentration. In order to eliminate the increase in $\mathrm{Na}^{+}$concentration, the annealing mixture was desalted using Microcon Montage-PCR filter units prior to the polymerization/ligation reaction. Filtration of the annealing reaction through such a membrane significantly increased the yield of ccDNA even when the complementary strand synthesis was performed for $2 \mathrm{~h}$ (Figure 1, lanes 1, 2, 5, and 6 versus lanes 3, 4, 7, and 8). Exclusion membranes used for the desalting of the annealing reaction retain only molecules of high molecular weight so that ions and also non-annealed primers are not retained by the membrane. Consequently, the increased efficiency of the polymerization/ligation reaction may not only be due to the elimination of monovalent cations but also to the elimination of the excess of phosphorylated primers.

Increasing the macromolecular crowding by the addition of high concentrations of nonspecific polymer has been shown to improve the efficiency of a variety of biological reactions (15). For example, the addition of high concentrations of PEG in the reaction buffer increases the T4 DNA ligase efficiency by several orders of magnitude (16) and the binding efficiency of T4 DNA polymerase to DNA (17). The addition of PEG to a final concentration of $7.5 \%$ greatly increased the yield of ccDNA produced during the polymerization/ligation reaction (Figure 1, lane 1-4 versus lanes 5-8). These results confirm that macromolecular crowding can significantly increase the efficiency of the polymerization/ ligation reaction.

In the conditions described above, the conversion of ssDNA 
molecules to ccDNA was incomplete, and further purification of the ccDNA was required. The ccDNA can easily be separated from the other unwanted DNA conformations because it migrates as supercoiled DNA in an aga- rose gel containing $0.3 \mu \mathrm{g} / \mathrm{mL}$ ethidium bromide and, once separated, ccDNA can be extracted from the agarose. Several methods are available for the elution of DNA from agarose, and it was necessary to define the procedure that would give the highest yield of DNA recovery. For this purpose, $10 \mu \mathrm{L}$ samples of the same polymerization/ligation were run in an agarose gel and different procedures for the extraction of ccDNA were compared (Table 1). The
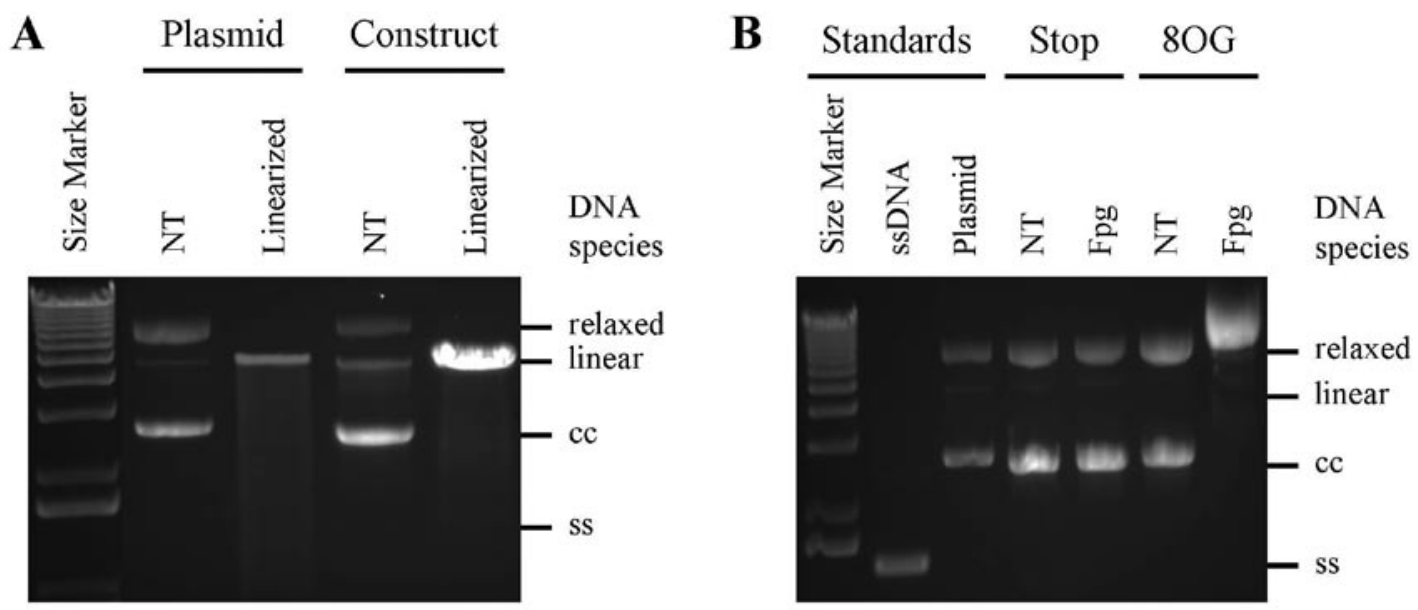

Figure 2. Verification of the eluted closed circular DNA (ccDNA). (A) pBESTluc-f1-Stop and eluted ccDNA were digested with BamHI (linearized) or not digested (NT). (B) Stop and 8OG constructs were treated with formamidopyrimidine (Fapy)-glycosylase (Fpg) or not treated (NT). As standards, ssDNA and nonlinearized dsDNA of the pBESTluc-f1-Stop plasmid were run simultaneously. The same amount of total DNA was loaded into each lane. (A and B) DNA size markers (1 Kb Plus DNA Ladders) were included in the far-left lanes. The electrophoretic mobility positions of single-stranded (ss), covalently closed circular (cc), linear and relaxed duplex DNA species are indicated. 8OG, 8-oxoguanine.

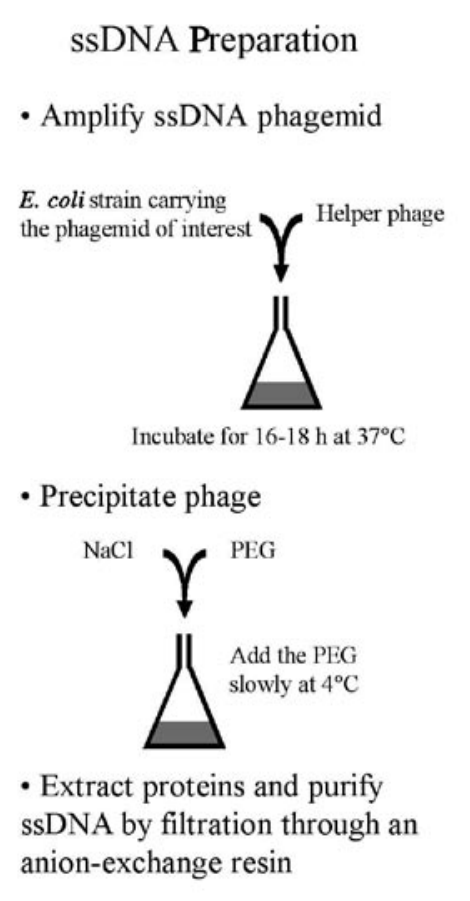

\section{Second-Strand Synthesis}

- Anneal ssDNA with a

$5^{\prime}$ phosphorylated primer

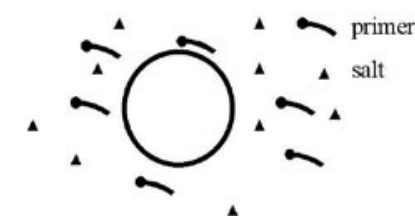

- Remove salts and excess of primers

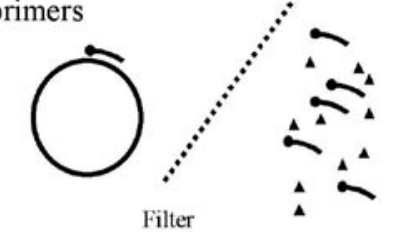

- Perform synthesis and ligation reaction

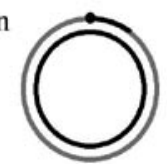

\section{ccDNA Purification}

- Run low melting point agarose gel containing ethidium bromide

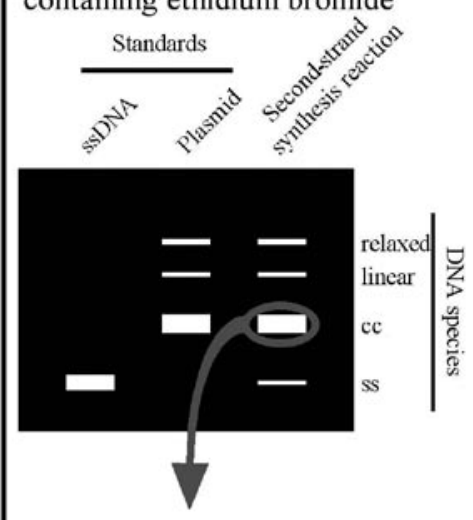

- Excise the band corresponding to ccDNA conformation

- Digest agarose with $\beta$-agarase and precipitate DNA

Figure 3. Critical steps for the generation of double-stranded DNA vectors containing site-specific base modifications. The generation of closed circular (cCDNA) vectors containing site-specific base modifications is achieved in three steps, which include the production of single-stranded DNA (ssDNA), the in vitro synthesis of the complementary strand containing a site-specific base modification, and the purification of the closed circular double-stranded DNA (dsDNA). E. coli, Escherichia coli; PEG, polyethylene glycol. 
most efficient procedure is the digestion of low melting point agarose with $\beta$-agarase and subsequent DNA precipitation with ethanol. In addition to its relative efficiency, this method has several advantages because the extracted DNA is of good quality (Table 1 , see $\mathrm{A}_{260} / \mathrm{A}_{280}$ ) and can be easily scaled up or down.

To confirm that the eluted DNA is a closed circular molecule, we digested it at a unique restriction site to linearize it. As shown on Figure 2A, it is clear that the eluted DNA can be linearized to give a DNA fragment migrating to a position equivalent to the linearized pBESTluc-f1-Stop plasmid. Nonetheless, it appears that the eluted DNA is not completely in the closed circular form because migration of this DNA in an agarose gel reveals one major band migrating equivalent to the eluted ccDNA and two minor bands corresponding to the relaxed and linear form. The generation of these minor bands is mainly due to the generation of singlestrand breaks during the elution and the storing of DNA. These DNA breaks are randomly distributed along the DNA molecule so that they should not have any influence on the studies made with these synthetic ccDNAs. However, if a pure ccDNA is required, one can purify it through a cesium chloride gradient or digest the eluted DNA with T7 exonuclease, which will efficiently degrade the relaxed and linear DNA and will not degrade the ccDNA (data not shown).

To ascertain that the abovedescribed construction method is useful for the study of translesion synthesis during transcription or replication in vivo, the $80 \mathrm{OG}$ construct was generated following the same procedure. Neither detectable decreases in the yield of ccDNA production nor decreases in transformation efficiency (from $10^{7}$ to $10^{8}$ transformants $/ \mathrm{mL} / \mu \mathrm{g}$ DNA) were observed when the polymerization/ligation reaction was primed with an 8OG-containing primer. To confirm the presence of $80 \mathrm{OG}$ in the resulting construct, the synthetic ccDNA was treated with FPG to introduce a nick in the $80 G$ ccDNA. The conversion of ccDNA to the relaxed conformation appeared to be complete in the case of the 8 OG construct whereas only a very small amount of the stop construct was converted (Figure 2B). This result indicates that $8 \mathrm{OG}$ construct molecules contain the desired DNA lesion at the targeted position.

The different steps for the production of synthetic ccDNA containing a DNA lesion at a specific position (depicted in Figure 3) were optimized to achieve relatively good yields. We are now extensively using this improved procedure, and from a single polymerization/ligation starting with $40 \mu \mathrm{g}$ ssDNA, we routinely obtain about $15 \mu \mathrm{g}$ synthetic ccDNA. The obtained DNA amount is sufficient for bacterial transformation and even for mammalian cell transfection. Furthermore, this procedure can easily be scaled up or down and does not require the use of expensive laboratory materials. The possibility of producing a large amount of ccDNA containing a lesion at a targeted position with this procedure should be very useful for the study of DNA and, especially, RNA polymerase translesion events in vivo and how they may be affected by the genetic background of the cell.

\section{ACKNOWLEDGMENTS}

We thank Christiane Dohet for valuable technical advice. This work was supported by National Institutes of Health grant nos. CA78622 and ES11163 (to P.W.D.).

\section{COMPETING INTERESTS STATEMENT}

The authors declare no competing interests.

\section{REFERENCES}

1.Doetsch, P.W. 2002. Translesion synthesis by RNA polymerases: occurrence and biological implications for transcriptional mutagenesis. Mutat. Res. 510:131-140.

2.Friedberg, E.C., R. Wagner, and M. Radman. 2002. Specialized DNA polymerases, cellular survival, and the genesis of mutations. Science 296:1627-1630.

3.Tornaletti, S. and P.C. Hanawalt. 1999. Effect of DNA lesions on transcription elongation. Biochimie 81:139-146.

4.Holmquist, G.P. 2002. Cell-selfish modes of evolution and mutations directed after transcriptional bypass. Mutat. Res. 510:141-152.
5.Weiner, M.P., G.L. Costa, W. Schoettlin, J. Cline, E. Mathur, and J.C. Bauer. 1994 Site-directed mutagenesis of double-stranded DNA by the polymerase chain reaction. Gene 151:119-123.

6.Lasken, R.S., D.M. Schuster, and A. Rashtchian. 1996. Archaebacterial DNA polymerases tightly bind uracil-containing DNA. J. Biol. Chem. 271:17692-17696.

7.You, H.J., A. Viswanathan, and P.W. Doetsch. 2000. In vivo technique for determining transcriptional mutagenesis. Methods 22:120-126.

8.Horsfall, M.J., A. Borden, and C.W. Lawrence. 1997. Mutagenic properties of the T-C cyclobutane dimer. J. Bacteriol. 179:28352839.

9.Zoller, M.J. and M. Smith. 1983. Oligonucleotide-directed mutagenesis of DNA fragments cloned into M13 vectors. Methods Enzymol. 100:468-500.

10.Kunkel, T.A. 1987. Oligonucleotide-directed mutagenesis without phenotypic selection, $\mathrm{p}$. 8.1.1-8.1.6. In F.M. Ausubel, R. Brent, D.D. Moore, J.G. Seidman, J.A. Smith, and K. Struhl (Eds.), Current Protocols in Molecular Biology. Wiley Interscience, Boston.

11.Bregeon, D., Z.A. Doddridge, H.J. You, B. Weiss, and P.W. Doetsch. 2003. Transcriptional mutagenesis induced by uracil and 8-oxoguanine in Escherichia coli. Mol. Cell 12:959-970.

12.Kunkel, T.A. 1985. Rapid and efficient sitespecific mutagenesis without phenotypic selection. Proc. Natl. Acad. Sci. USA 82:488492.

13.Hayashi, K., M. Nakazawa, Y. Ishizaki, and A. Obayashi. 1985. Influence of monovalent cations on the activity of T4 DNA ligase in the presence of polyethylene glycol. Nucleic Acids Res. 13:3261-3271.

14.Chiu, C.S., K.S. Cook, and G.R. Greenberg. 1982. Characteristics of a bacteriophage T4induced complex synthesizing deoxyribonucleotides. J. Biol. Chem. 257:15087-15097.

15.Ellis, R.J. 2001. Macromolecular crowding: obvious but underappreciated. Trends Biochem. Sci. 26:597-604

16.Pheiffer, B.H. and S.B. Zimmerman. 1983. Polymer-stimulated ligation: enhanced blunt- or cohesive-end ligation of DNA or deoxyribooligonucleotides by T4 DNA ligase in polymer solutions. Nucleic Acids Res. 11:7853-7871.

17.Zimmerman, S.B. and B. Harrison. 1987. Macromolecular crowding increases binding of DNA polymerase to DNA: an adaptive effect. Proc. Natl. Acad. Sci. USA 84:18711875

Received 3 May 2004; accepted 16 June 2004.

Address correspondence to Paul W. Doetsch, Division of Cancer Biology, and Department of Radiation Oncology, Emory University School of Medicine, 1510 Clifton Road, Atlanta, GA, USA. e-mail: medpwd@emory.edu 